\title{
The Contourlet Domain Comic Zero Watermarking Algorithm based on Discrete Cosine Transform and Singular Value Decomposition
}

\author{
De $\mathrm{Li}^{1}$, Haifeng $\mathrm{Shi}^{1}$ and JongWeon $\mathrm{Kim}^{2 *}$ \\ ${ }^{1}$ Department of Computer Science, Yanbian University, Yanji, China \\ leader1223@ybu.edu.cn,shi.haif@163.com \\ ${ }^{2}$ Department of Intellectual Property, Sangmyung University, Seoul, Kbrea • \\ jwkim@smu.ac.kr,*jwkim@smu.ac.kr \\ Abstract
}

Through to analyze the characteristics of the digital cartoon image, proposed a based on discrete cosine transform (DCT) and singular value decomposition (SVD) combined contourlet transform with zero watermarking algorihm, the algorithm first for image contourlet transform and then make the low-frequency subband DCT and SVD decomposition again after operation block, the largest singular value as the image feature extracting for zero watermarking structure. In order to improve the security of the algorithm, introducing into visual cryptography processing the watermark inage, the zero watermarking features obtained and secret sharing for the logic operation is zero watermarking characteristics of the final value.. The simulation results show that the olgorithm has strong robustness.

Keywords: Cartoon image, Contourlet transform, Singular value decomposition, Visual Cryptography

\section{Introduction}

With the information times continuously, the development of the Internet becomes more and more rapidly, also becomes more and more popular in people's daily lives, more people began to publish their digital yyorks uploaded to the network, not only rich the multimedia information but also makes the multimedia data storage, replication, transmission becomes more and more easily. Accompanied by the emergence of a wide variety of digital works, cartoon network because of its concise and interesting style is becoming more and more popular, cartoon image is different from the general digital image and image content includes both traditional image content, at the same time equipped with a witty humor or meaningful words. At the same time because digital comic image and other cartoon images with less amount of data, shows advantages of high quality and easy retrieval, so it has become the electronic book, personal digital assistants (PDA) and handheld computers and other consumer electronics more and more popular media form on [1]. For the same reason, along with digital comic images and other cartoon images on the network widely exists and transmission, so that the intellectual property protection of image and integrity authentication has become a fundamental issue. It can be said that the Internet in convenient for people to exchange, enrich people's life at the same time, also proposed the stern test of copyright protection of cartoon image and similar to the multimedia works. This paper in view of the cartoon image put forward a based on the discrete cosine transform and singular value decomposition combined contourlet transform with zero watermarking algorithm, meanwhile, the direction of the successful application and communication of visual cryptography will

* Corresponding Author 
introduced into the algorithm in this paper, guarantees the robustness and invisibility of the watermark under the premise of a certain extent, improve the security of the algorithm.

The traditional digital watermark method generally can be divided into two categories: frequency domain watermarking method $[2 \sim 6]$ and spatial domain watermarking method [7, 8]. These methods of embedding watermark are modified the information of image the spatial information or the frequency domain information to embed watermark information, in order to do not let the human find traces of man-made changes, many methods based on the use of HVS [9] (human visual system) visual mask. Methods such as literature [10] have proposed an adaptive image watermarking method using HVS visual mask. This method for visual mask in a certain of extent solved the contradiction between the intellectuality and robustness of the watermark. But with the visual mask makes the embedding process complexity, the consumption of computing time is too long, it is not conducive to the practical application. In addition, because of the digital image watermarking technology is the traditional watermark information will be embedded into the carrier image to show imâges copyrigh, it will cause image distortion in a certain of extent, and after the attacked, the watermark extraction will be difficulty. According to these problems, Wen Quan 111] proposed a view of zerowatermarking which can use the important image features to construct watermark information, but not modify these image features. This method that does not modify any data of the original image but to construct the secret information by using the important features of the original image and then the secret information can be registered to the intellectual property data information base to proye the original earrier image copyright called "zero watermarking". Here defined zero watermarking method is a new digital watermark method, zero watermarking technology to solke the invisible digital watermarking of the contradiction between the perception and robustness.

\section{Related Work}

This paper based on the study of cartoon image, attributed to both the general characteristics of the linage, and the characteristics of the text image, therefore, we introduce the contourlet transform, singular value decomposition and manipulate visual cryptography and so on in the algorithmane then we are introduced one by one.

\subsection{Contourlet Transform}

Contourlet transform is also known as contour wavelet transform, it is an extension of wavelet transform, it's composed of two parts that decomposition by Laplacian Pyramid and directional filter banks, therefore contourlet transform also called Pyramid directional filter banks. Contourlet transform has good multi-resolution, fine features, such as localization and orientation than wavelet is more suitable for capture the high dimensional singularity characteristics of the information. It's can only use a few coefficients efficiently represent the smooth contour and the contour smooth is the important features of the image, but also has a similar to the curvelet transform of the anisotropy scaling relation [12].

First of all, the original carrier signal will be decomposed into low frequency and high frequency subband by the way of tower. The low frequency subband is original carrier signals a low-pass sampling approach and the high frequency subband is the difference between original image and the low frequency subband. Then, directional filter banks will function in the high frequency subbands, have an arbitrary scale high-pass subband by multiple directions. Because directional filter banks are not suitable for processing the low frequency sub-band part, so the low frequency part after removing the carrier before using DFB, otherwise the low-frequency information will fall in each directional subband. By 
decomposition of low frequency subband part for many times, you can achieve carrier multiresolution decomposition of multiple directions. Contourlet transform is a kind of sparse representation method of carrier can represent the vector contour and texture fully. The support interval is rectangular structure, along with the change of decomposition scale change.

In the traditional watermarking algorithm, the watermark embedded options selected in the contourlet coefficient. Own characteristic of contourlet transform can know, tower of decomposed high frequency subband is not through high-pass filtering, but acquired through the original carrier signal and the low frequency of a low-pass filter with subtraction. Therefore, embedding the watermark into the high-frequency band, modifying coefficients of high frequency subbands, will also affect the low frequency subband. The reason is thatin the inverse contourlet transform reconstruction, watermark will be scattered to the low rrequency part of the carrier.

To sum up, different transform domain has its own adxantages and disadvantages, such as discrete cosine transform domain has good shaped features. discrete wavelet transform domain has characteristic of multi-resolution analysis contourlet domain has multiple directions and so on. If you can combine the advantages of different transform domain, the robustness of the watermark algorithm can be improved to a certain extent.

\subsection{Singular Value Decomposition}

Singular value decomposition (SVD) is) a kind of (Im)portant matrix decomposition, and linear algebra is a generalization of patrix analysis of normal matrix unitary diagonalization. Theorem: if $\mathrm{A} \in \mathrm{R}[\mathrm{m} \times \mathrm{n}]$, then there exists an orthogonal matrix:

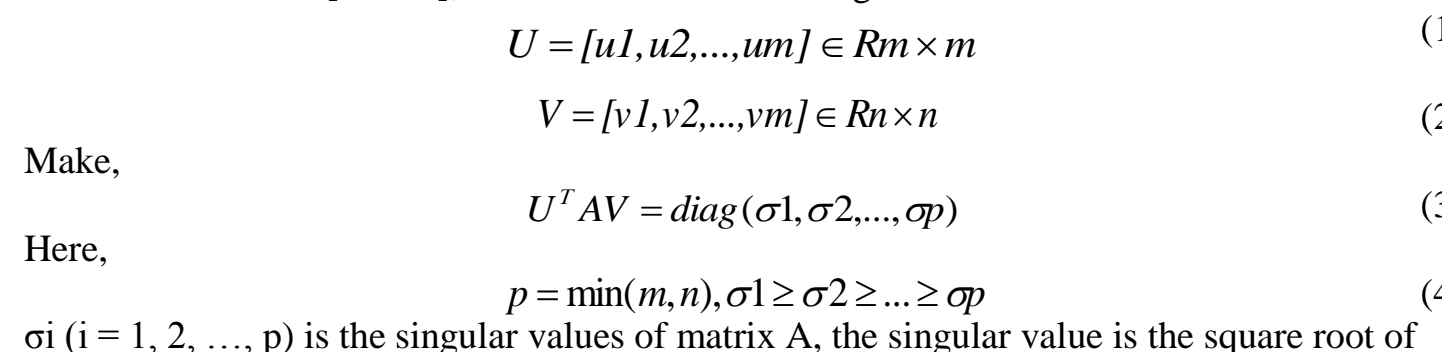

$\sigma \mathrm{i}(\mathrm{i}=1,2, \ldots, \mathrm{p})$ is the singular values of matrix $\mathrm{A}$, the singular value is the square root of [13] matrix $\mathrm{A}^{\mathrm{H}} \mathrm{A}$ and matrix $\mathrm{AA}^{\mathrm{H}}$ value.

Singular values can be used as a kind of characteristic has been applied in image processing and recognition, and its main theoretical basis is the singular value decomposition characteristics, was the following properties:

(1) The stability of the SV feature vector: because the only corresponding relation between the original image and its SV feature vector, so you can use the SV feature vector to describe two-dimensional images. When there is small changes in the image gray level, the SV feature vector whether there will be a big change, if there will not be a big change, it is stable. SVD perturbation analysis indicate that the SV feature has good stability, so it's different to image noise, image illumination conditions caused by image gray level change has characteristic of less sensitive. This feature to relax the requirement for image preprocessing. The experimental results confirmed that the SV feature is not sensitive to noise.

(2) The SV feature vector of transposed invariance: if do the image matrix transpose operations, the SV feature vectors are not changed.

(3) The SV feature vector of therotation invariance: rotating operations to image, the SV feature vectors are not changed.

(4) The SV feature vector of displacement invariability: replacement operations to image matrix, the SV feature vectors are not changed. 
(5) The SV feature vector of image transform invariance: for any kind of image feature extraction, which extract features with algebraic and geometric invariance. For SV features, these properties to ensure that it has the invariance, which is based on the theory as a kind of algebraic feature of image using it.

\subsection{Visual Cryptography}

Visual cryptography [14] is a new image sharing technology, it is the combination of secret sharing and digital image. Visual cryptography by secret sharing algorithm encodes a secret image into several shares, and distributed to each participant. When the set of participants meet agreed to restore conditions, only let the sharing of participants direct superposition can recover the secret image. Because the visual cryptography secret recovery process is simple, so the research emphasis focuses on secret sharing algorithm. This paper adopts the secret sharing algorithm base matrix as the core, make sure the safety of visual passy/ord schemes and comparative conditions, thus base matrix design becomes the key to risual cryptography scheme in this paper. Naor and Shamir proved that the minimure pixel expansion $(n, n)$ degree is $2 \mathrm{n}-1$ and give the method to construct he basis matrix that is B0 (share matrix white pixels) consists of all the Hamming weight as even cofumn vector, B1 (share matrix black pixels) consists of all the Hamming weight as odd column vector. Bludno [15] proved that $(2, \mathrm{n})$ threshold scheme of minimumpixel expansion to meet $C(m,\lceil m / 2\rceil) \geq n$ about the minimum values of $\mathrm{m}$, whet base matrix design, B0 consists of $\mathrm{n}$ identical Hamming weight for $[\mathrm{m} / 2\rceil$ row vector and $B 1$ by different Hamming weight for $[\mathrm{m} / 2\rceil$ row vector. This paper use $(2,2)$ scheme and the desigh basís matrix is $B_{0}=\left[\begin{array}{ll}1 & 0 \\ 1 & 0\end{array}\right]$ and $B_{1}=\left[\begin{array}{ll}1 & 0 \\ 0 & 1\end{array}\right]$ superposition of two shared Copy about T1 and T2 will be restore the image S'. To be sure, direct superposition shared copy of the Sucan fully recover the original image S. In addition to different base matrix, the corresponding recovery function $\mathrm{R}$ is different.

\section{The Zero Watermarking Algorithm Design}

This paper adopts the netyork common cartoon image as the carrier image experiment, the cartoon image by contourlet transform to get the low frequency subband and followed by the DCT and SVD method of combining and processing the low frequency subband to get the features of the priginal cartoon image value. The cartoon image is different from the general digital image form the content, cartoon image includes not only the content of the image, but also contains some text content; from the characteristics, not only have the basic feature of general inage, but also carrying the corresponding characteristics of text image. Therefore, we first contourlet transform to the cartoon image. Contourlet transform is a kind of sparse representation method of carrier, can represent the vector contour and texture fully, the image content comic image and text are processed through many decomposition makes the corresponding data with multiple resolution of direction. To get the data after combining DCT and SVD method for processing. Discrete cosine transform has shaped the characteristics, singular value decomposition can enhance the invisibility of the watermark and ensure that the watermark has good robustness, and the zero watermarking method instead of the traditional digital watermarking method, for the cartoon image, contourlet transform can respectively according to the image content and good processing text content, while at the same time can through the both very good together by zero watermarking algorithm, but also ensure the use value of the cartoon image, so the algorithm is applicable to cartoon image. 


\subsection{Watermark Embedding}

The embedded watermark algorithm in this paper is not the traditional sequence code or a bit stream, but the watermark will be treated as a binary image processing and hidden, which makes the watermarking information contained more rich and intuitive. Watermarking embedded algorithm is described as follows, detailed process shown in Figure 1:

Step 1: The original color image is converted to grayscale images, then based on the gray image for the following processing;

Step 2: Processing the grayscale images of Contourlet transform and extract the low frequency subband;

Step 3: The low frequency subband $2 \times 2$ blocks DCT transformation and extracted the lowfrequency component;

Step 4: The extract low-frequency component again for $2 \times 2$ blocks SVD decomposition and extract the decomposition of the largest singular value;

Step 5: For binarization of extracting the largest singular yalue, form the characteristic sequence;

Step 6: Read the original watermarking image, using visual cryptography, get two share of Share1 \& Share2;

Step 7: Feature sequence value and Share1 or Share2 have the associated logical operations to get the zero watermarking characteristics of the final value. In this step we can also be carried on corresponding processing to Share such as a piece of data to extract only the particular logic operations with characteristic sequence value, in order to further improve the security of the algorithm itself.

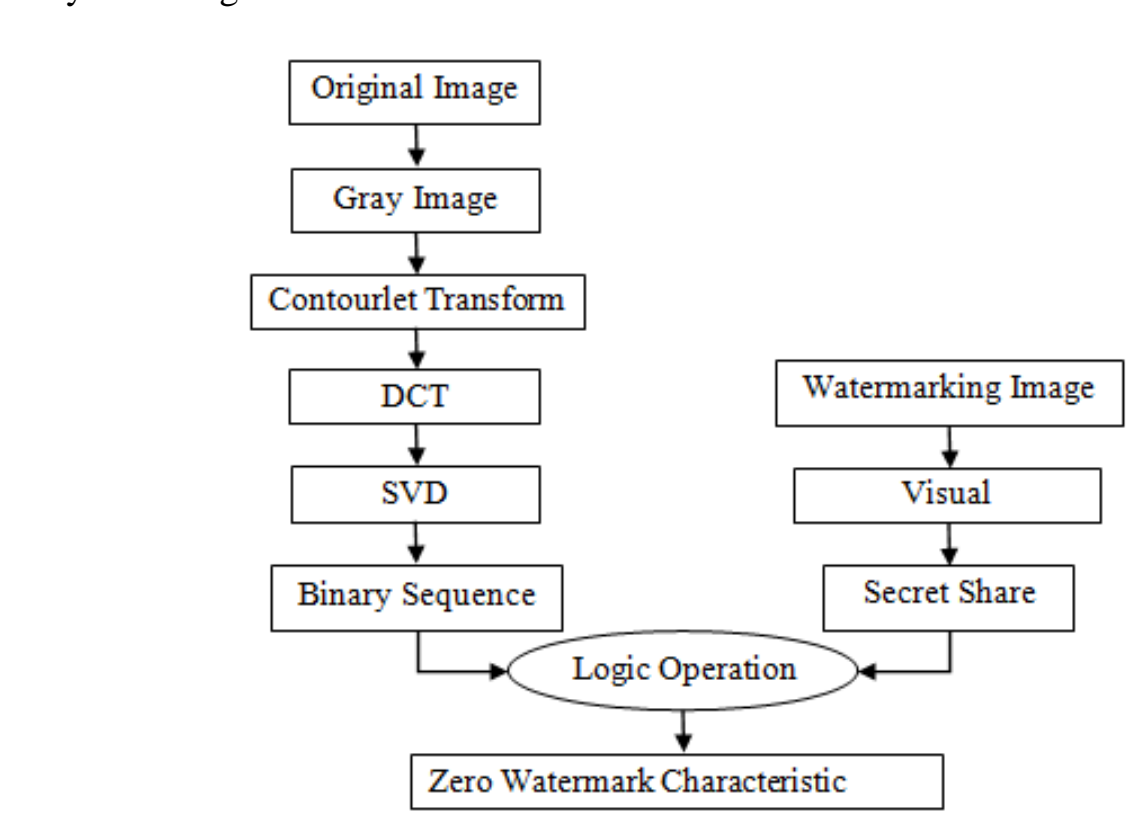

Figure 1. Watermark Embedding Process Flow Diagram

In the above algorithm, select only the largest singular value causes as the image features of the original carrier image to construct zero watermark information is through the handling of the original image and after block SVD decomposition of singular value and through JPEG compressed images through a comparison between SVD decomposition of singular value, found that only the largest singular value is relatively stable, and the rest of the singular value 
because of its itself value is small, change is opposite bigger, construct zero watermark is very difficult to recover after JPEG compression after the watermark information.

\subsection{Watermark Extracting}

By the concept of the zero watermark, in construct zero-watermarking characteristics of the final value, which should be registered to the intellectual property data information base to prove the original carrier image copyright ownership, which means that at the end of the watermark embedding process, retain the information will only end up with zero watermark characteristics, as well as in the visual cryptography scheme of watermark image is to share relevant position information data extraction. The watermark extraction algorithin is described as follows, detailed process shown in Figure 2:

Step 1: To the original color image into grayscale image conversion attack experiment, after the attack to image for the following processing;

Step 2: Contourlet transformation of the attacked image, extract the low-frequency band;

Step 3: The low frequency subband for $2 \times 2$ block DCT and extracted the low-frequency component;

Step 4: To extract the low-frequency component again for $2 \times 2$ block SVD and extract the decomposition of the largest singular value;

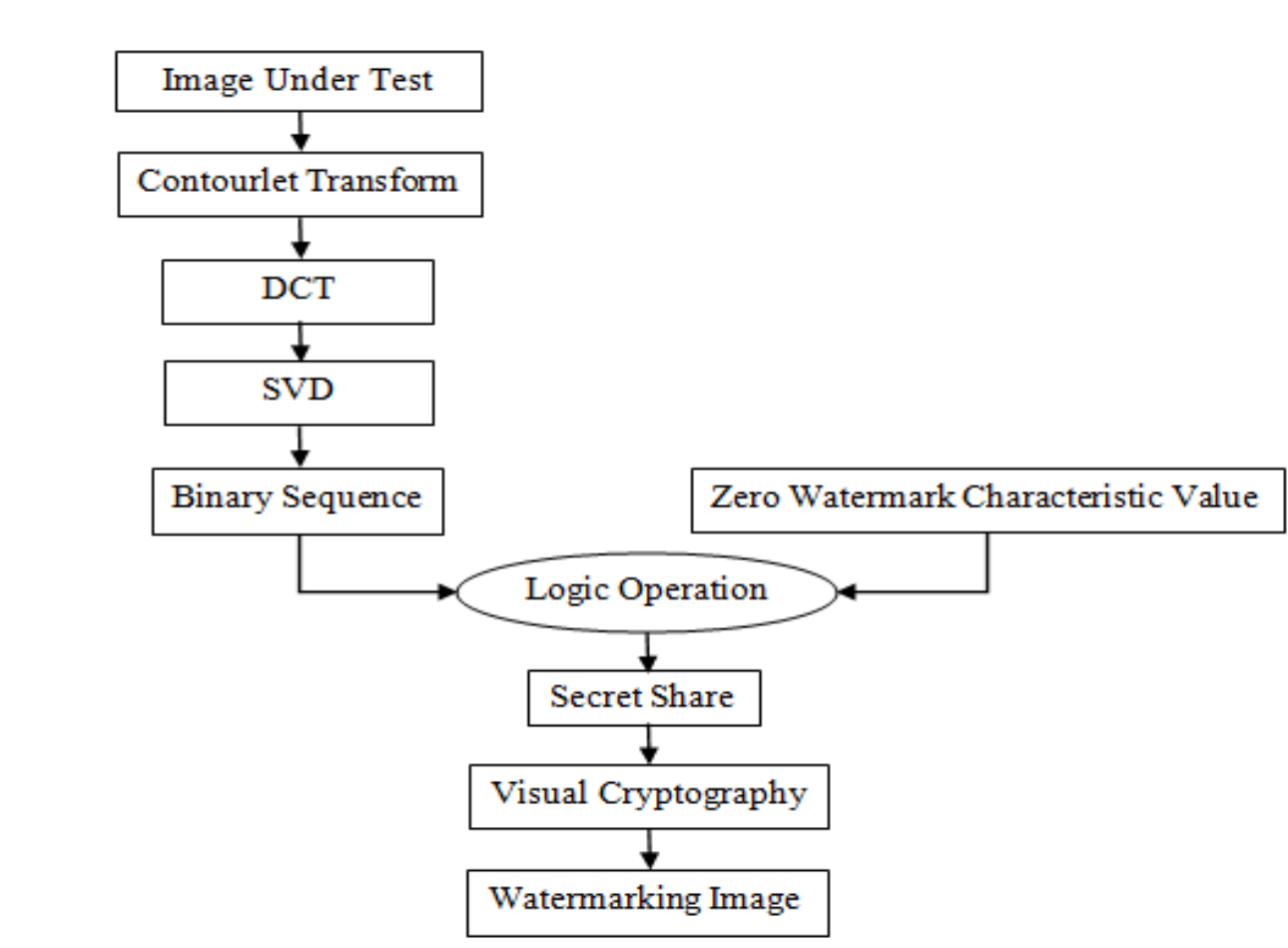

Figure 2. Watermark Extracted Process Flow Diagram

Step 5: For extracting the largest singular value of binarization form feature sequence;

Step 6: Read the zero watermark characteristic values that get to the watermark embedding process and restoring the sharing data of visual cryptography logic operation with characteristic sequence;

Step7: The sharing data through visual cryptography secret operation restore a watermark image. 
The above described Step 7, the sharing of the secret operation must be corresponding to the seventh step in the process of watermark embedding operation, otherwise not only cannot recover the watermark image but also increase the complexity of the algorithm itself.

In this algorithm, in order to increase the algorithm's security, we have a visual cryptography shared copy again for processing. We can be in two shares select a piece of data that obtained with image feature sequence same size then logic operation. Therefore, if we want to recover the original watermark, should know the share that in secret sharing algorithm of visual cryptography, also need to know selected data in the share. In the process of recovery of the watermark image, steps like above, just need to modify the Step 6 and Step 7.Because we save the zero watermarking characteristics final value after constructed the zero watermark, so when we recovery the watermark image the original image to features of attack after the obtained values and eventually zero watermarking characteristic value with the logic operation, so that the correct visual cryptography share to recover the ofiginal ${ }_{\text {watermark }}$ image.

\section{The Experimental Results and Analysis}

In order to validate the performance of the algorithm, this paper carried out the attack experiments in Matlab R2011b. Including noise,cropping, scaling, filtering, compression and other attacks.

Papers randomly selected from a pair of 512 x 512 size of the color cartoon image experiment, as shown in figure 3. Watermark image as "the yanbian university computer science" of English acronyms "YBCS with size, $32 \times 32$, as shown in Figure 4.

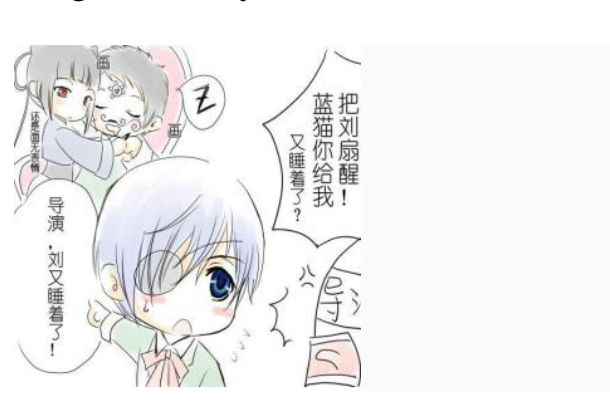

Figure 3. Original Image

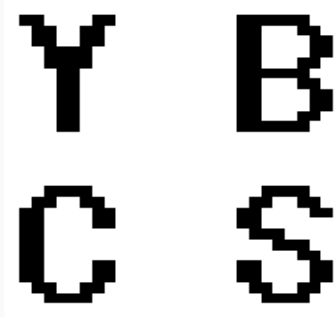

Figure 4. Watermark Image

Because of the robustness and imperceptibility of watermark is a very important factor to measure performance, and the zero watermarking algorithms, imperceptibility, so only through the $\mathrm{NC}$ and the BER value to measure the robustness of watermarking algorithm. As mentioned in the watermark embedding algorithm, first of all need to the original carrier image, the color image is converted to a grayscale image, as shown in Figure 5.

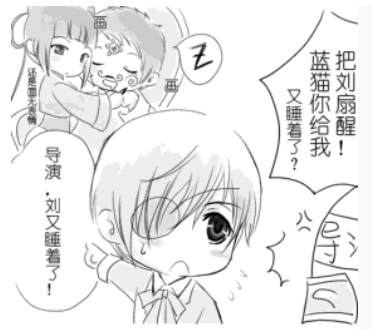

Figure 5. Grayscale Image

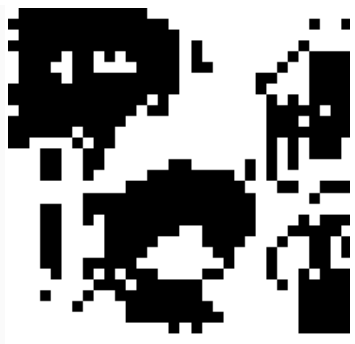

Figure 6. Binaryzation Array w 
After contourlet transform and DCT decomposition and SVD decomposition combined with the original image, get the original carrier image binarization sequence $\mathrm{w}$, as shown in Figure 6. This paper to the original watermark image used the visual cryptography get two Shared that is Share1 and Share2, respectively as shown in Figure 7, as shown in Figure 8, if the visual cryptography inversion directly on the shared portions of Share and Share2, change a other words, untreated directly to share a secret operation, can directly recover the original watermark image, as shown in Figure 9. At the end of the watermark embedding process, and ultimately get characteristics of the zero watermark, as shown in Figure 10.

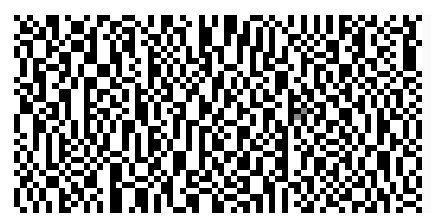

Figure 7. Share1

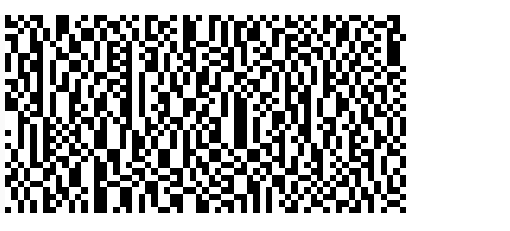

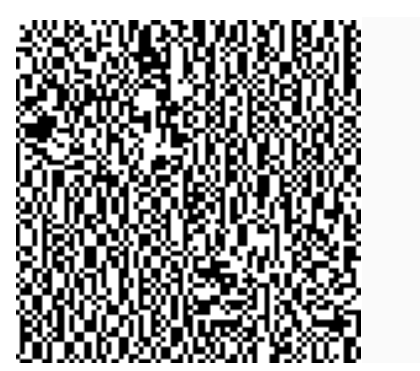

Figure 9. Eigenvalue of Zero Watermarking
Figure \& Share2

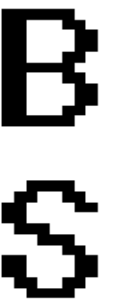

Figure 10. Lossless Recover Watermark Image

This paper images to test for a varjety of common attack experiment, in order to evaluate the performance of the watermarking algorithm. In general, the extract (restore) embedded watermark and the original vatermark similarity (NC) is 1 , bit error rate (BER) is 0 that can shown the algorithm compretely accurate to extract the watermark.

To verify the algorithm robustness against noise attacks, chose Gauss noise and salt-pepper noise attack experiment, the experimental results are shown in Table 1.

$\begin{array}{lll}\text { Attacked image } & & \text { Tarble 1. Recovered Results of Noise Attacked } \\ \text { Gauss } \\ \text { noise } \\ \text { attack }\end{array}$




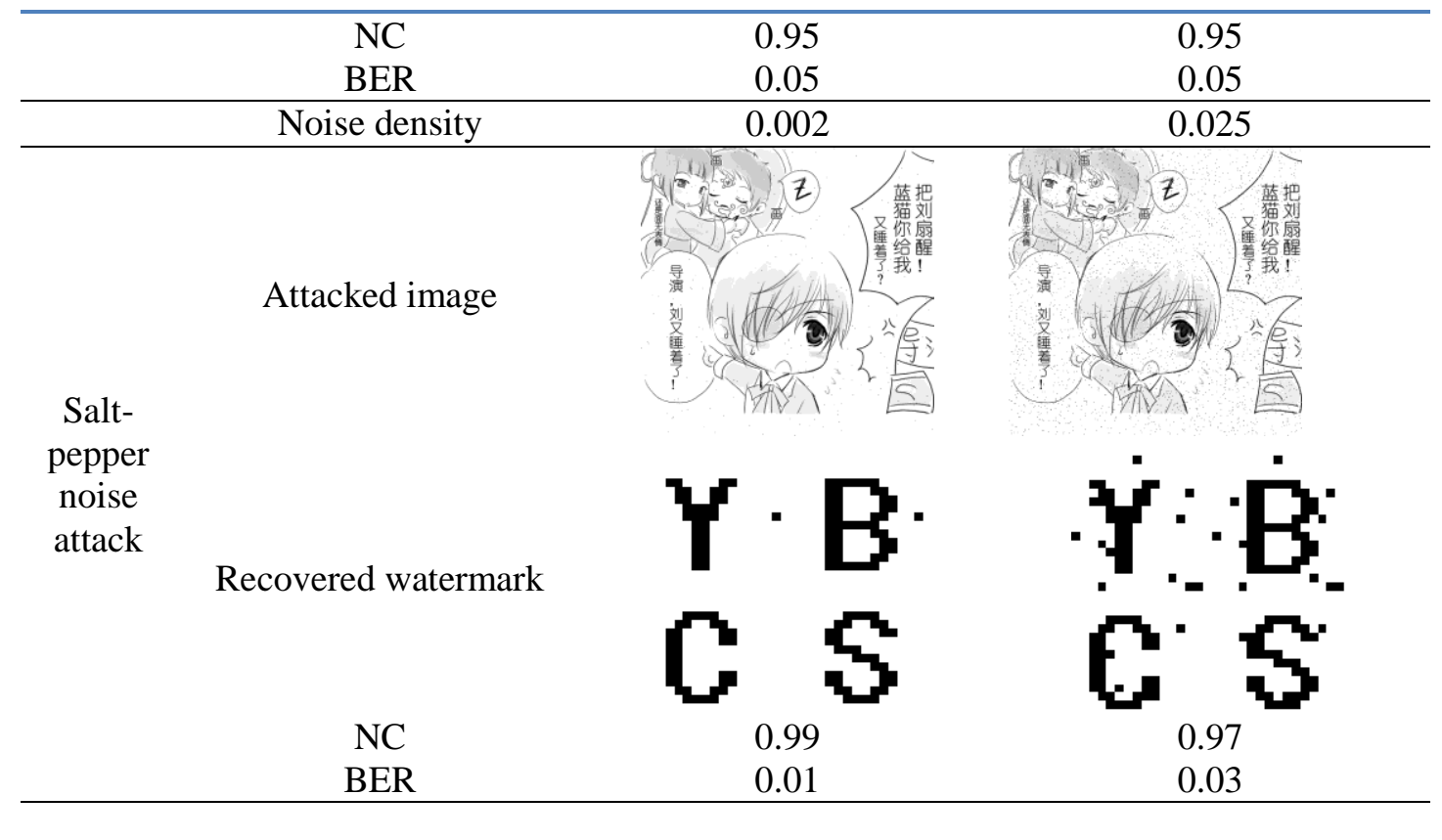

As can be seen from Table 1, when applying a larger degree of noise attack, and even affect the sensory effects of the orighnal image, we dan still get a recognizable image watermark to prove the ownership of the digital works, which have the effect of copyright protection.

To verify the algorithm robustness in resisting scaling attack, zoom in and out of the attack experiment was carried out respectively, the results are shown in Table 2.

\section{Table 2. Recovéred Results of Scaling Attacked}

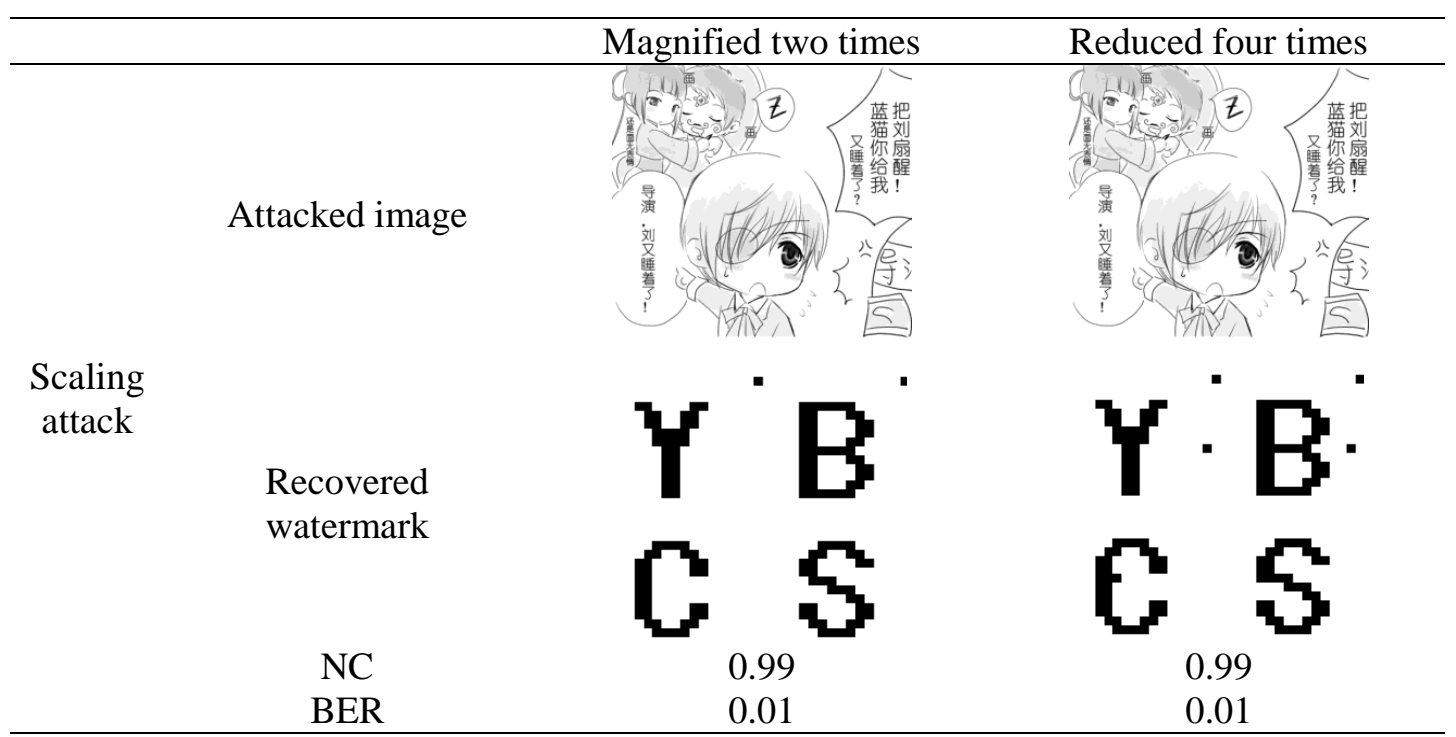

As can be seen from Table 2, whether to reduce or enlarge the operation of the original image, both on the sensory effects of the original image is small, easy to get a clear image watermark to prove the ownership of the digital works, which have the effect of copyright protection. 
To verify the algorithm robustness in filtering attack, choose the four neighborhood filtering and 8 neighborhood filtering attack experiment, the experimental results as shown in Table 3.

\section{Table 3. Recovered Results of Filtering Attacked}

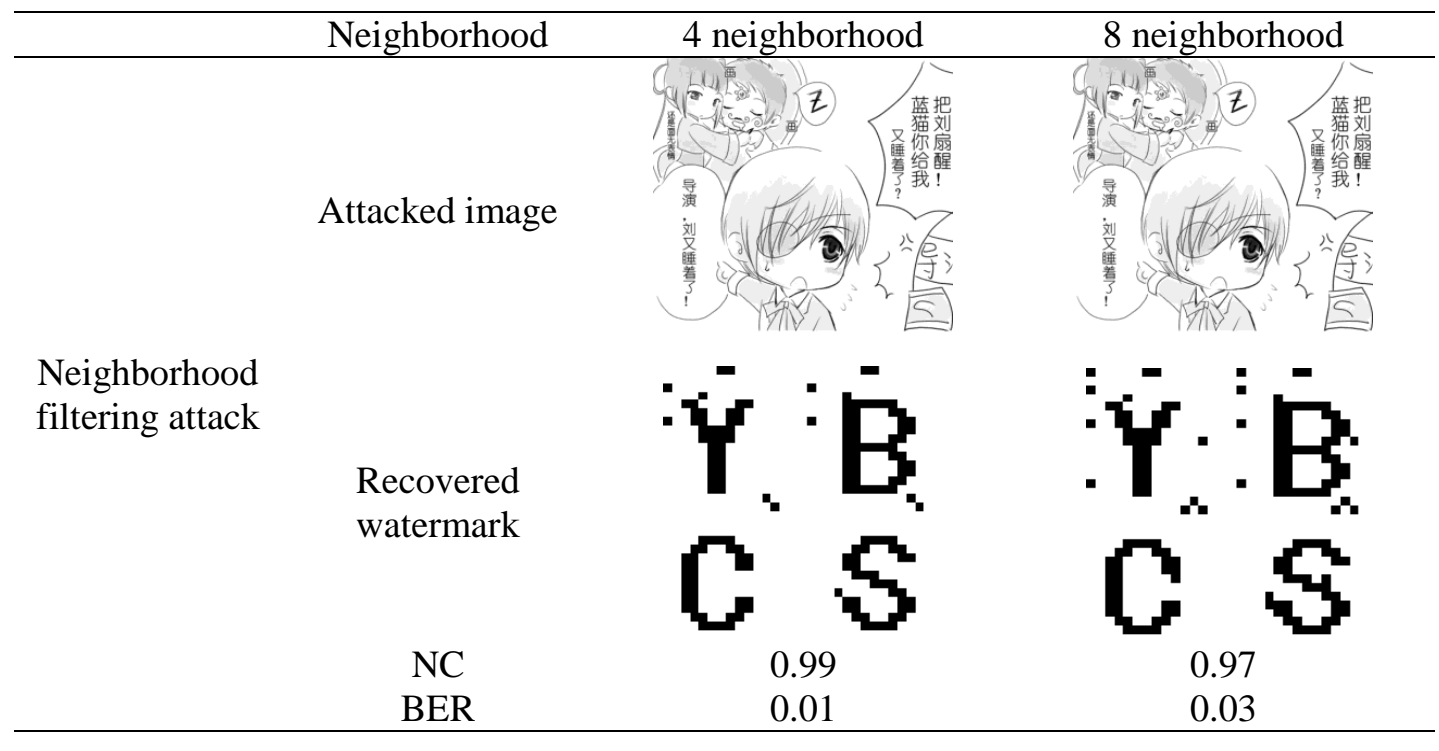

As can be seen from Table 3 , neighborhood filtering the original image, the sensory effects is small, easy to get a clear image watermark to prove the ownership of the digital works, which have the effect of copyight protection.

To verify the robustness in terms of against cropping attack, choose the upper left and lower right cropping attack experiment, the experimental results as shown in Table 4.

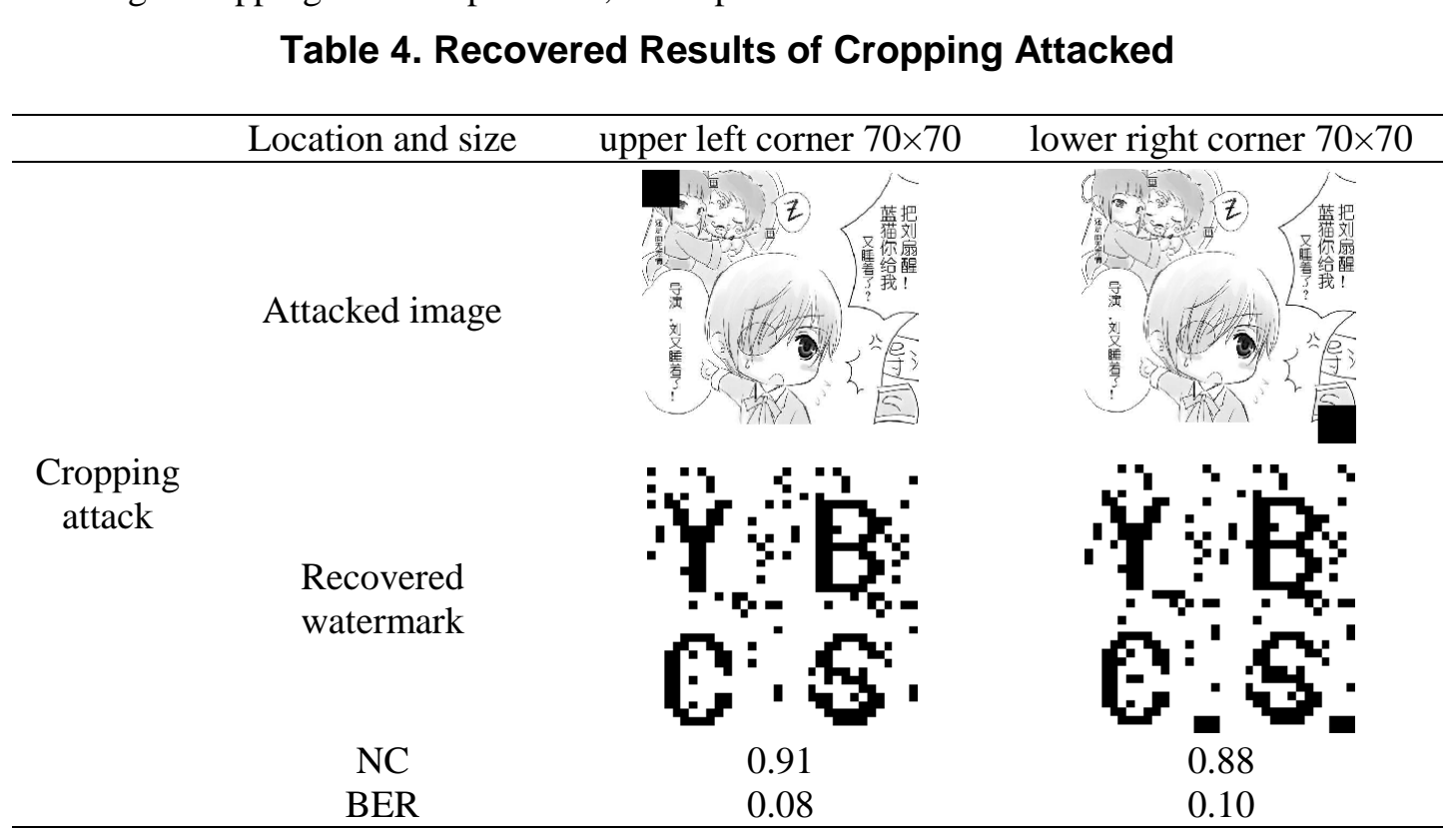

As can be seen from Table 4, the original image in the upper left corner or the bottom right hand corner cutting operation, the influence is bigger, seriously affected the image of the 
sensory effects, but can still recovering from a recognizable image watermark to prove the ownership of the digital works, which have the effect of copyright protection.

To verify the algorithm robustness in resistance to compression attack, choose JPEG compression attacks experiment, experimental results as shown in Table 5.

Table 5. Recovered Results of Compression Attacked

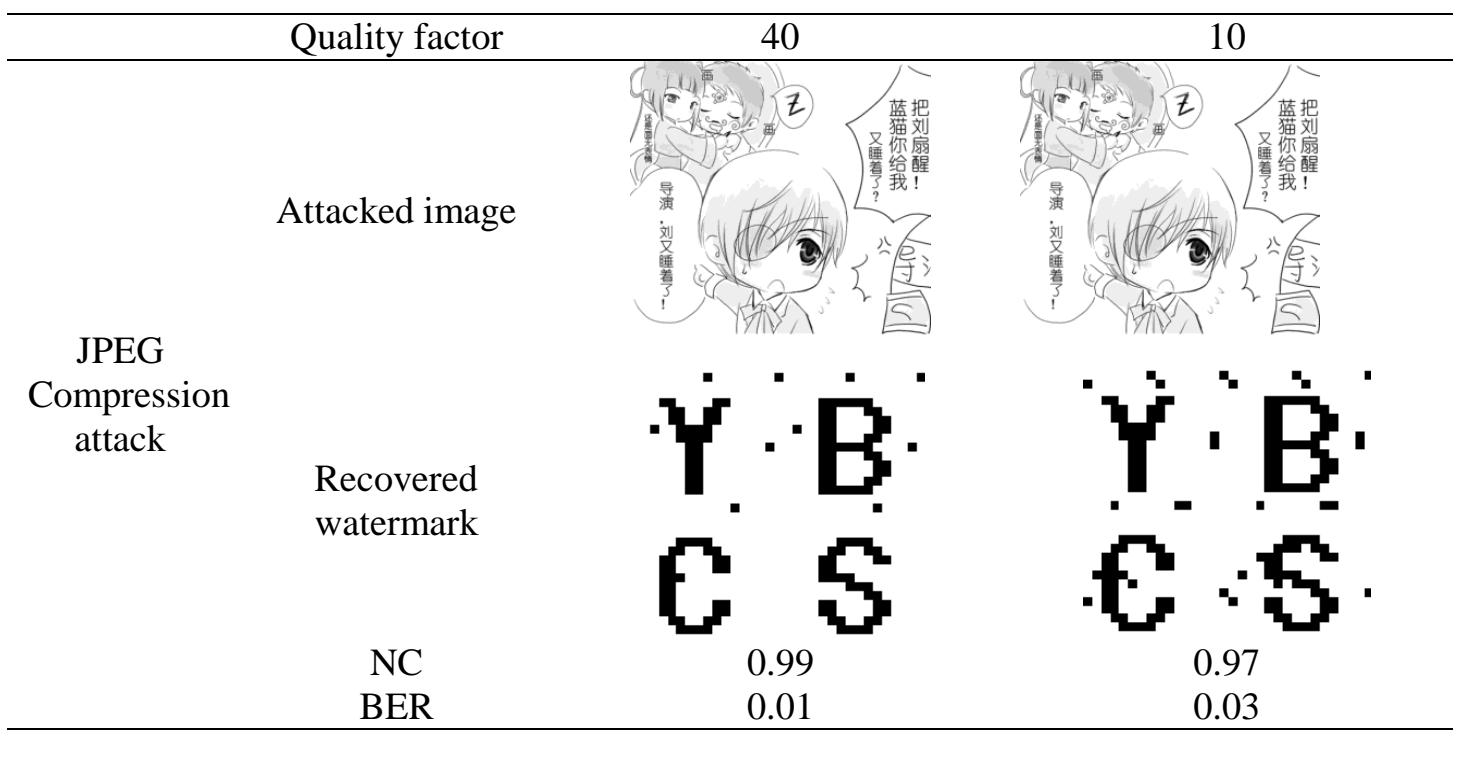

As can be seen from Table 5, when the PPEG compression quality factor is reduced ceaselessly less sensory effect on the original image and we can still get a recognizable image watermark to prove the ownership of the digital works to protect the copyright.

By the attack experiment results dan be seen that the algorithm of this paper is to resist noise attack, scaling attack, neighborhood filtering, compression attack, the effects on the original image the sensory effects were small, good results have been achieved, along with visual cryptography was jntroduced in the algorithm, while guaranteeing the original image fully restored, effectively reduce the pixel expansion, but does not increase the computational complexity of the recovery process conditions, effectively improve the safety performance of the algorithm, intesrated concluded in this paper the algorithm performance is stronger.

Through further research of algorithm, the similarity of NC value and the error rate of BER shows the algorithm for cropping attack resistance is weak, the greater influence on the recovery of watermark image. The reason for the initial, the original contourlet transform, through two discrete decomposition of low frequency subband, brought together most of the basic features of the original image and it's beneficial to resist geometric attacks. And then Methods DCT and SVD combined, it's can enhance the robustness of the watermarking algorithm in some extent, but before the transformation we obtain the low-frequency bands were $2 \times 2$ block, thereby reducing the basic features of the original image between the elements of the correlation, and may produce the block effect, coupled with singular value decomposition on SVD values of two values processing makes the basic features of the original image data has changed to some extent let the algorithm in the paper for the weak ability to resist cropping attack. 


\section{Conclusion}

This paper based on the digital cartoon image, through further research to the contourlet transforms has been get a zero watermark algorithm based on the DCT and SVD combination of contourlet transform. The algorithm first two layers of the discrete contourlet transform to the image and then low frequency subband decomposition operation block after DCT decomposition combined with SVD decomposition to extract the maximum singular value decomposition as the feature of image to construct the zero-watermark, in order to made a good balance between the robustness and invisibility of the request use visual cryptography to encrypt the watermark image operation, not only without increasing the complexity of the algorithm but also greatly improve the security of the algorithm itself.

\section{Acknowledgements}

This research project was supported by the Ministry of Culture, Sports and Tourism (MCST) and the Korea Copyright Commission in 2014.

\section{References}

[1] X. H. Ma, "Digital watermarking method for image copyright protection of digital comics", The first session of the build a harmonious human environment conference (HHME 2005) proceedings. Beijing, (2005), pp. 864-868

[2] J. W. Huang, Y. Q. Shi and W. D. Cheng, DCT domain image watermarking embedding strategy and algorithm", Journal of electronics, vol. 28, nø. 4, (2000), pp. 57-60.

[3] X. M. Niu, Z. M. Lu and S, H. Sun, "Digital watermarking technology based on multi-resolution decomposition", Journal of electronics, vol. 28, no. 8 , (2000), pp. 10-12.

[4] R. Z. Liu and T. N. Tan, "The digital image watermarking method based on singular value decomposition", Journal of electronics, vol.29 nd. 2, (2001), pp. 168-17.

[5] M. Barni, F. BartolinP and A.Piva, "Improved Wavelet Based Watermarking Through Pixel-Wise Masking [J]", IEEE Transactions on lmage Procéssing, vol. 10, no. 5, (2001), pp. 783-791.

[6] Y. X. Wang and C. G. Wang "A nem digital image zero-watermarking algorithm based on Contourlet transform", Journal of Y Yanshan University, vol. 34, no. 6, (2010), pp. 528-531.

[7] F. J. Zeng and A. M. Zhou, "Image zero-watermarking algorithm based on contourlet transform and singular value decomposition", Computer applications, vol. 28, no.8, (2008), pp. 2033-2035.

[8] H. Y. Zhao, K. Liu and A. J. Li, "A Digital image zero watermarking algorithm based on wavelet transform", Coal technology, vol. 30, no. 11, (2011), pp. 164-168.

[9] T. Y. Ye, "A robast zero watermarking algorithm based on variance in singular value decomposition domain", Journal of Photon., vol. 16, no. 06, (2011), pp. 231-236.

[10] W. J. Wang and B. He, "A zero watermarking algorithm in multi-discrete cosine transform and singular value decomposition", Computer and digital engineering, vol. 29 no. 06, (2011), pp. 132-137.

[11] Q. Wen, G. F. Sun and S. X. Wang, "The concept and application of zero watermark", Journal of electronics, vol. 31, no. 2, (2003), pp. 1-3.

[12] L. Q. Chen, X. Y. Sun, M. Lu and C. Shao, "Contourlet watermarking algorithm based on Arnold scrambling and singular value decomposition”, Journal of Southeast University, vol. 28, no. 4, (2012), pp. 386-391.

[13] F. Y. Zhang, H. L. Quan, L. Y. Lin and Q .Q. Qin, "The Robust Digital Watermarking Algorithm based on singular value decomposition in Contourlet domain", Computer application and research, vol. 29, no. 4, (2012), pp. 1402-1408.

[14] B. Yu and Z. X. Fu, "The research of lossless share visual cryptography", Journal of China Institute of communications, vol. 34, no. 3, (2013), pp. 165-170.

[15] C. Bludno, A. D. Santis and Dr. Stinson, "Graph Decomposition and secret sharing schemes [J]", Journal of Cryptology, vol. 8, (1995), pp. 39-64. 


\section{Authors}

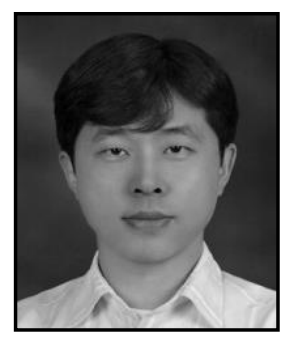

De Li received the Ph.D. degree from Sangmyung University, major in computer science in 2005. He is currently a professor of Dept. of Computer Science at Yanbian University in China. He is also a Principal Researcher at Copyright Protection Research Institute, Sangmyung University. His research interests are in the areas of copyright protection technology, digital watermarking, and digital forensic marking.

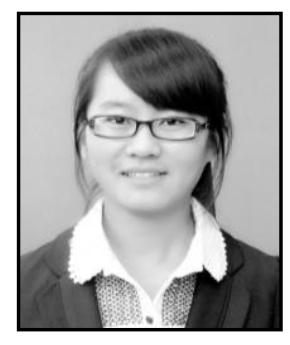

HaiFeng Shi is a postgraduate, major in Information Security, now studying at Yanbian University in China. Her research interests are in the areas of copyright protection technology, informationsecurity, digital watermarking and digital forensic marking.

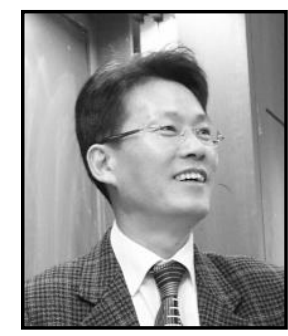

JongWeon Kim received the Ph D. degree from University of Seoul, major in signal processing in 1995. He is currently a professor of Dept. of Intellectual Property at Sangmyung Univensity in Korea. He has a lot of practical experiences in the digital signal processing and copyright protection technology in the irstitutional, the industrial, and academic environments. His research interests are in the areas of copyright protection technology, digital rights management, digital watermarking, and digital forensic marking. 
International Journal of Multimedia and Ubiquitous Engineering Vol. 9, No. 10 (2014)

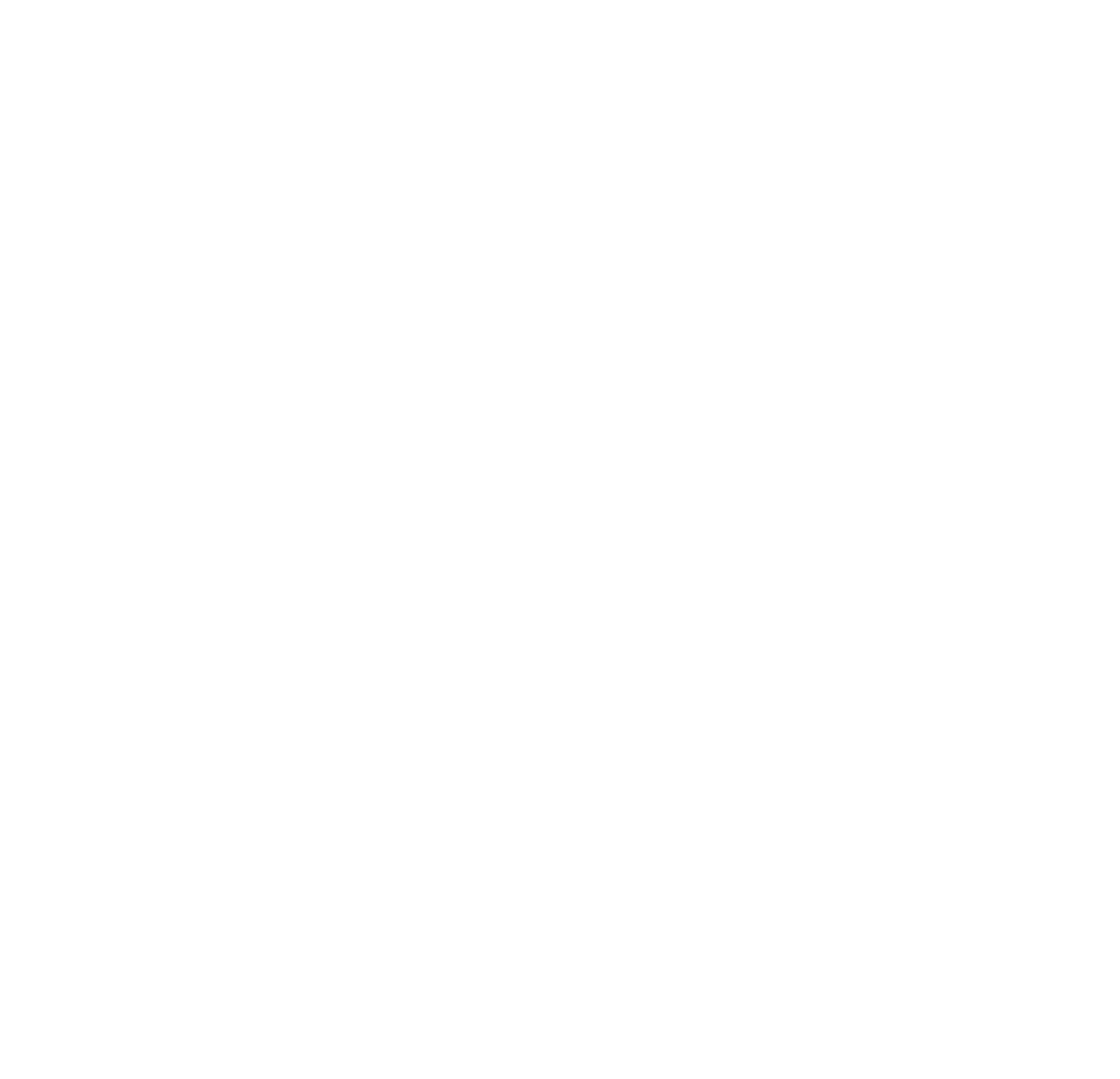

